\title{
EFEITO DOS BLOQUEADORES DO RECEPTOR DE ANGIOTENSINA NA INIBIÇÃO DAS CITOCINAS IL-17A E IL-17F EM PACIENTES COM ATRITE REUMATOIDE
}

\author{
K. A. MATIAS ${ }^{1}$, P. R. G. CARDOSO ${ }^{1}$, A. T. DANTAS ${ }^{3}$, C. D. L. MARQUES ${ }^{3}$, M. J. B.
} M. REGO ${ }^{1}$, A. L. B. P. DUARTE ${ }^{3}$, I. R. PITTA ${ }^{2}$ e M. G. R PITTA ${ }^{1}$

${ }^{1}$ Laboratório de Imunomodulação e Novas Abordagens Terapêuticas - Universidade Federal de Pernambuco

${ }^{2}$ Núcleo de Pesquisa em Inovação Terapêutica - Suely Galdino - Universidade Federal de Pernambuco

3 Ambulatório de Reumatologia, Hospital das Clínicas da Universidade Federal de Pernambuco

E-mail para contato: katherineaquinom@gmail.com

RESUMO - O objetivo deste trabalho foi avaliar a atividade imunomoduladora in vitro dos bloqueadores do receptor da angiotensina II, losartana e valsartana, e do inibidor da enzima conversora de angiotensina, enalapril, sobre a produção das citocinas IL-17A e IL-17F, em PBMCs provenientes de pacientes portadores de AR. 19 pacientes com AR, selecionados aleatoriamente, compuseram a amostra. Na avaliação in vitro, as PBMCs foram estimuladas ou não com anticorpo monoclonal anti-CD3 e anti-CD8, na presença de enalapril, losartana e valsartana a $100 \mu \mathrm{M}$, combinados ou não ao metotrexato a

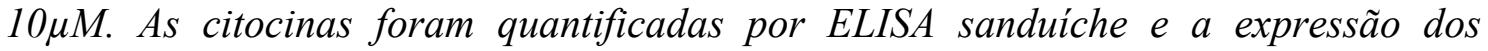
resultados das variáveis contínuas foi feita pelas médias e desvios padrão. Os sobrenadantes de cultura de IL-17F nenhuma associação estatisticamente significativa foi observada. Os níveis de IL- $17 F$ apresentaram uma queda na produção da citocina estatisticamente significativo na losartana a 100 $\mu \mathrm{M}[273,14(1239,83$ - 15,62)] $(p=$ 0.0107) comparado com a condição estimulação [436,00 $(3836,50-15,62)]$ e também deste medicamento em associação com o metotrexato a $10 \mu M[261,71(1548,17$ - 15,62)] $(p=0.0023)$ quando comparado com a condição só de estímulo. Conclui-se que a losartana é o fármaco anti-hipertensivo mais eficiente na supressão da expressão de IL$17 F$, apresentando possível ação sobre as células Th17.

Palavras-chave: DCV, células T, AT1, inflamação, hipertensão.

ABSTRACT - The objective of this study was to evaluate the in vitro immunomodulatory activity of angiotensin II receptor blockers, losartan and valsartan, and the angiotensin converting enzyme, enalapril, on production of IL-17A and IL-17F cytokines in PBMCs of patients with RA. 19 RA patients, randomly selected, composed the sample. In the in vitro evaluation, PBMCs were stimulated or not with anti-CD3 and anti-CD8 monoclonal antibodies, in the presence of enalapril, losartan and valsartan at 100 $\mathrm{M}$, combined or not with methotrexate at 10 $\mathrm{MM}$. The cytokines were quantified by sandwich ELISA and the expression of the continuous variables' results was done by median and standard deviations. IL-17F culture supernatants had no statistically significant association. IL- 
$17 F$ levels showed a decrease in statistically significant cytokine production in Losartan at $100 \mu M[273.14(1239.83-15.62)](p=0.0107)$ compared to the stimulated condition $[436.00(3836,50-15,62)]$ and also of this drug in combination with $10 \mu M$ methotrexate $[261,71(1548,17-15,62)](p=0.0023)$ when compared to the only-stimulus condition. In conclusion, losartan is the most effective antihypertensive drug in the suppression of IL-17F expression, presenting possible action on Th17 cells.

Key-words: CVD, T-cells, AT1, inflammation, hypertension.

\section{INTRODUÇÃO}

A artrite reumatoide (AR) é uma doença inflamatória crônica caracterizada pelo dano articular progressivo que leva a incapacitação grave e a mortalidade prematura e atinge cerca $1 \%$ da população (Symmons e Gabriel, 2011). Das mortes prematuras, 50\% são atribuídas a doença cardiovascular (DCV), como a doença isquêmica do coração, a insuficiência cardíaca congestiva e a hipertensão arterial sistêmica (HAS) (Kozera et al. 2011) e a incidência de morte cardíaca súbita é duas vezes maior nos portadores de AR que na população em geral (Symmons e Gabriel, 2011).

Evidências recentes demonstram o papel da inflamação no desenvolvimento da DCV e diversos mecanismos inflamatórios e imunológicos parecem estar envolvidos na etiopatogênese da HAS, interagindo em sua maior parte com o sistema renina-angiotensina aldosterona (SRAA) (Silva et al. 2013). A Ang II ativa várias funções celulares e vias de sinalização moleculares relacionadas com dano, inflamação e fibrose (Capettini et al., 2012). Nos anos recentes, vários estudos indicam a expressão de AT1, principal receptor alvo de Ang II e a ação da Ang II na produção de IL-17A a partir de células T (Dinh et al. 2014; McMaster et al. 2015; P. Wenzel et al. 2011).

As células T também são um importante elemento na patogênese da artrite reumatoide, em relação ao desbalanço de suas variantes. Th17 é uma subpopulação caracterizada de células T que induzem a produção de IL-17. A IL-17 conduz a síntese de vários fatores-chave como TNF- $\alpha$, IL-1 $\beta$, IL-6, IL-8, proteína quimiotáctica de monócitos-1 (MCP-1), prostaglandina E2 e promove a quimiotaxia de neutrófilos e a produção de granulócitos, diferenciação de osteoblastos e contribuindo para o dano da cartilagem e dos ossos (Furst e Emery 2014). Segundo Lee e colaboradores (2013) a IL-17 também age promovendo a proliferação de sinoviócitos e resgatando os sinoviócitos da morte apoptótica através da via STAT3. A família de citocinas IL-17 é um grupo recentemente identificado de citocinas, que inclui seis membros: IL- 17A, B, C, D, E (IL-25) e F (Azizi et al. 2013). De acordo com Furst e Emery (2014), tanto a IL-17A como a IL-17F ativam um complexo de receptor heterodimérico composto por subunidades de proteínas IL-17RA e IL-17RC. A IL-17F regula a expressão do gene pró-inflamatório por uma via de sinalização semelhante, mas não idêntica, a da IL-17A, downstream de IL-17RA e IL-17RC.

Este trabalho tem por objetivo avaliar a atividade imunomoduladora in vitro dos bloqueadores do receptor da angiotensina II (BRA's), losartana e valsartana, e do inibidor da enzima conversora de angiotensina (ECA), enalapril, sobre a produção das citocinas da via Th17, IL-17A e IL-17F, em PBMCs provenientes de pacientes portadores de AR. 


\section{MATERIAIS E MÉTODOS}

\subsection{Voluntários}

Este trabalho foi aprovado pelo comitê de ética do Centro de Ciências da Saúde da UFPE (parecer de $\mathrm{n}^{0}$ 1.486.212). A avaliação clínica e convite de participação dos voluntários, além da coleta de material biológico, foram realizadas no ambulatório de artrite reumatoide do Serviço de Reumatologia do HC-UFPE. A amostra do trabalho foi composta por 19 indivíduos com AR. Amostras de sangue periférico (SP) dos voluntários (3 homens e 16 mulheres), foram coletados em $18 \mathrm{~mL}$ de $\mathrm{SP}$ em tubos contendo heparina.

\subsection{Critérios de Inclusão e Exclusão}

Critérios de inclusão: idade acima de 18 anos; ser portador de AR, diagnosticado de acordo com os critérios do American College of Rheumatology (ACR) ou de acordo com os critérios do ACR/EULAR de artrites iniciais; estar em acompanhamento regular no ambulatório de reumatologia do HC-UFPE.

Critérios de exclusão: não consentimento em participar do estudo; estar em uso regular de anti-hipertensivos que atuam sobre o SRAA (inibidores da ECA, BRA's, betabloqueadores); estar em uso de medicamentos imunobiológicos ou imunossupressores; não possuir outras comorbidades reumatológicas ou que afetem o sistema imune, como canceres.

\subsection{Citotoxicidade}

Para avaliar a citotoxicidade do Enalapril, Losartana e da Valsartana foi realizado o teste do MTT (3-(4,5- dimetiltiazol-2- il)-2,5- difenil brometo tetrazólico). As células foram incubadas a $37^{\circ}$ e $5 \%$ de $\mathrm{CO} 2$ durante 48 horas. Foi adicionada a solução de MTT $(20 \mu \mathrm{l})$ $(0,5 \mathrm{mg} / \mathrm{mL})$ e as placas retornaram a incubadora de $\mathrm{CO} 2$ por mais 3 horas. Em seguida, foram adicionados $120 \mu \mathrm{L}$ da solução de SDS (Dodecil Sulfato de Sódio) 20\% para dissolução do precipitado. A leitura das placas foi realizada, 24 horas depois, em um espectrofotômetro a um comprimento de onda de 570nm.

\subsection{Cultura de PBMCs}

As células mononucleares do sangue periférico (PBMCs) foram isoladas por centrifugação com Ficoll PaqueTM Plus (GE Healthcare Bio-Sciences). As PBMCs isoladas foram cultivadas (10e6 células/ml) em meio RPMI 1640 (Gibco) suplementado com LGlutamina, 10\% de Soro Bovino Fetal (Lonza), $10 \mathrm{mM}$ de HEPES (4-(2-hydroxyethyl)-1piperazineethanesulfonic acid) (Gibco) e $200 \mathrm{U} / \mathrm{mL}$ de Penicilina/Estreptomicina (Gibco). Elas foram estimuladas ou não com anti-CD3 e anti-CD28, na presença de enalapril, losartana e valsartana, nas doses definidas. Estas células foram cultivadas em estufa de $\mathrm{CO} 25 \%$ a $37^{\circ} \mathrm{C}$ por $48 \mathrm{~h}$ e após isso o sobrenadante celular foi coletado e estocado a $-30^{\circ} \mathrm{C}$. O Metotrexato foi utilizado como medicamento padrão. Foi feito também a associação dos anti-hipertensivos com o metotrexato com a finalidade de similar o padrão de tratamento do paciente com AR e HAS.

Para a quantificação de IL-17A e IL-17F foi realizada a técnica do ELISA seguindo as recomendações do fabricante (EBiosciences - limite de detecção 3,9 e 15,6 pg/mL respectivamente). Utilizou-se sobrenadante de cultura celular para a detecção e avaliação das citocinas. 


\subsection{Análise Estatística}

O teste de Wilcoxon e T de Student foram utilizados para as análises estatísticas e $\mathrm{p}<$ 0.05 foi considerado estatisticamente significativo. Os valores são expressos em mediana, máxima e mínima para as dosagens de citocinas nos sobrenadantes e média \pm desvio padrão (DP) para os dados demográficos que não apresentarem porcentagens. Todas as análises estatísticas foram realizadas utilizando o GraphPad Prism ${ }^{\circledR}$ 6.01.

\section{RESULTADOS E DISCUSSÃO}

Um total de 19 pacientes participaram do estudo e preencheram 4 ou mais critérios do ACR para o diagnóstico da AR. A avaliação clínica foi realizada utilizando o DAS28 e o CDAI. Os dados clínicos e laboratoriais estão disponíveis na Tabela 1.

Tabela 1: tabela das características dos pacientes e suas ocorrências.

\begin{tabular}{|c|c|}
\hline $\begin{array}{c}\text { Características } \\
\text { Pacientes }(n=19)\end{array}$ & Dados \\
\hline Feminino $\mathrm{n}$ & 16 \\
\hline Masculino $\mathrm{n}$ & 3 \\
\hline Idade (anos), média (mín-máx) & $53(29-73)$ \\
\hline $\begin{array}{l}\text { Tempo de duração da AR (anos), média } \\
\text { (desvio) }\end{array}$ & $10( \pm 7,4)$ \\
\hline $\begin{array}{l}\qquad \text { DAS28 }(\%) \\
\text { Baixa atividade }(\leq 3.2) \\
\text { Moderada Atividade }(>3.2 \leq 5.1) \\
\text { Alta Atividade }(>5.1)\end{array}$ & $\begin{array}{l}9(48 \%) \\
7(37 \%) \\
3(15 \%)\end{array}$ \\
\hline $\begin{array}{l}\text { CDAI }(\%) \\
\text { Remissão }(\leq 2.8) \\
\text { Baixa atividade }(>2.8 \text { and } \leq 10) \\
\text { Moderada Atividade }(>10 \text { and } \leq 22) \\
\text { Alta atividade }(>22)\end{array}$ & $\begin{array}{l}3(15 \%) \\
6(31 \%) \\
3(15 \%) \\
7(37 \%)\end{array}$ \\
\hline $\begin{array}{ll} & \text { Tratamento }(\%) \\
\text { Prednisona } & \\
\text { Metotrexato } & \\
\text { Leflunomida } & \end{array}$ & $\begin{array}{c}12(63 \%) \\
14(72 \%) \\
3(15 \%)\end{array}$ \\
\hline \begin{tabular}{l}
\multicolumn{2}{c}{ Comorbidades (\%) } \\
Diabetes Mellitus \\
Obesidade \\
Osteoporose
\end{tabular} & $\begin{array}{l}1(5 \%) \\
1(5 \%) \\
1(5 \%)\end{array}$ \\
\hline VSH média (desvio) & $17( \pm 9,7)$ \\
\hline Fator Reumatóide (\%) & $19(100 \%)$ \\
\hline
\end{tabular}

Após a coleta de sangue, citotoxicidade dos medicamentos anti-hipertensivos de escolha Enalapril (E), Losartana (L) e Valsartana (V) em PBMCs de doadores voluntários sadios foi avaliada para confirmar a seletividade. Foram avaliadas também, cinco diferentes concentrações de dose $(10,25,50,75$ and $100 \mu \mathrm{M})$ em triplicata de dose e experimento. 
Nenhum dos medicamentos testados apresentou toxicidade seletiva para PBMCs, apresentando uma viabilidade superior a $98 \%$ em todas as condições.

Como a AR é uma doença mediada pelo desbalanço das subpopulações das células T descrito anteriormente, o estímulo das culturas de PBMCs foi feito com anticorpos monoclonais anti-CD3 e anti-CD28 a fim de estimular apenas as células T que possuem esses receptores. Na disposição da cultura, as células foram ou não estimuladas com os anti-CD3 e anti-CD28 na presença de Enalapril, Losartana e Valsartana a $100 \mu \mathrm{M}$. O metotrexato foi utilizado na sua dose ideal para cultura, ou seja, $10 \mu \mathrm{M}$. Além disso, também foi avaliado o co-tratamento do metotrexato junto aos anti-hipertensivos em outro poço de cultura.

Nenhuma associação estatisticamente significativa foi observada nos valores dosados de IL-17A dos sobrenadantes de cultura. Quando avaliado os níveis de IL-17F, observou-se uma queda na produção dessa citocina estatisticamente significativo na Losartana a $100 \mu \mathrm{M}[273,14(1239,83-15,62)](\mathrm{p}=0.0107)$ comparado com a condição estimulada com anti-CD3 e anti-CD28 [436,00 (3836,50 - 15,62)] e também deste medicamento em associação com o metotrexato a $10 \mu \mathrm{M}[261,71(1548,17-15,62)](\mathrm{p}=0.0023)$ quando comparado com a condição só de estímulo. Losartana $(100 \mu \mathrm{M})$ também obteve melhores resultados na diminuição dos níveis de expressão de IL-17F [273,14 $(1239,83$ - 15,62)] comparado com a droga padrão, o metotrexato a $10 \mu \mathrm{M}[737,60(2931,50-15,62)](\mathrm{p}=$ 0.0269), como observados nas Figuras 1 e 2.

Figura 1 - A imagem 1 mostra os níveis de IL-17A em cultura de PBMCs de pacientes portadores de AR nas condições Células (C), Células Estimuladas (CE), Metotrexato a $10 \mu \mathrm{M}$ (MTX), Enalapril (E), Losartana (L), Valsartana (V) a 100 $\mu \mathrm{M}$ cada e a associação do Enalapril, Losartana e Valsartana com o Metotrexato: (E/MTX; L/MTX; V/MTX respectivamente). Não foi observada nenhuma associação estatisticamente significativa. Os resultados são expressos em Mediana (Máxima - Mínima).

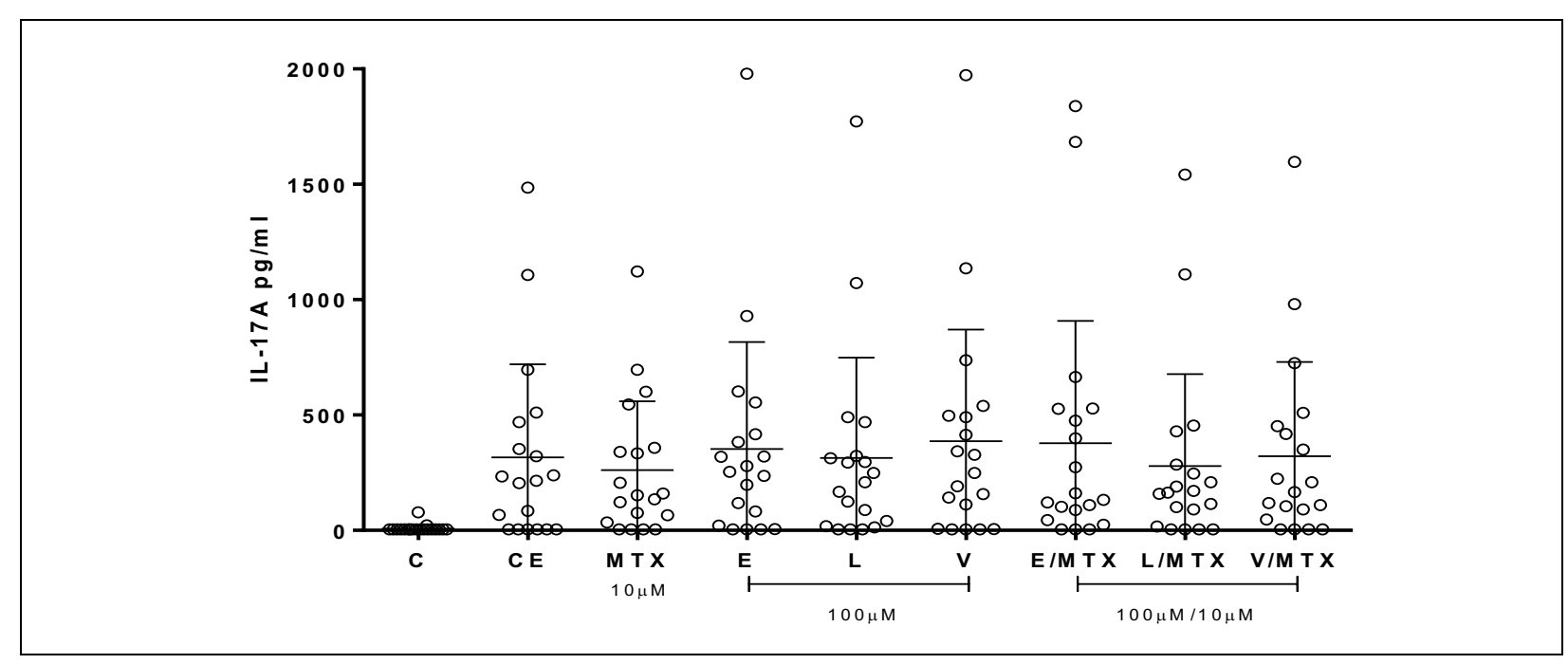


Figura 2 - A imagem 2 mostra os níveis de IL-17F em cultura de PBMCs de pacientes portadores de AR nas condições Células (C), Células Estimuladas (CE), Metotrexato a $10 \mu \mathrm{M}$ (MTX), Enalapril (E), Losartana (L), Valsartana (V) a $100 \mu \mathrm{M}$ cada e a associação do Enalapril, Losartana e Valsartana com o Metotrexato nas mesmas doses anteriormente utilizadas: (E/MTX; L/MTX; V/MTX respectivamente). Losartana se mostrou eficiente em reduzir os níveis de IL-17F em comparação com a condição tratada ( $\left.{ }^{*} \mathrm{p}=0.0107\right)$ e também quando comparada ao Metotrexato (\# $\mathrm{p}$ $=0.0023$ ). Além disso a associação da Losartana e o Metotrexato se mostra eficiente em reduzir os níveis de IL-17F comparado a condição de estímulo CE ( $p=0.0269)$. Os resultados são expressos em Mediana (Máxima - Mínima).



A redução significante da expressão da IL-17F sob o efeito da Losartana aponta para uma possível ação dessa droga sobre as células IL17-F. Essa hipótese é reforçada pela alta expressão da IL-17A, uma vez que esta é produzida não só pela Th17, mas também pela Th1 (Furst e Emery 2014). Outro importante ponto é que a Losartana, tanto administrada isoladamente quanto em associação ao Metotrexato, mostrou-se mais eficiente que o Metotrexato na dose padrão. Por sua vez, a Valsartana e o Enalapril não apresentaram ação efetiva em nenhuma das condições em que foram aplicados. Esses dados dirigem a hipótese que apesar de tanto a Losartana como a Valsartana serem ambos bloqueadores de angiotensina, sua ação anti-inflamatória em relação as ações da linhagem de células Th17 é divergente. Essa informação é importante para formular abordagens para o tratamento de um paciente com AR e apresentando quadro de hipertensão arterial.

\section{CONCLUSÃO}

Com base nos dados desse estudo conclui-se que a Losartana é o fármaco antihipertensivo com maior eficiência na redução da expressão da citocina pró-inflamatória IL-17F, e com provável ação sobre as células Th17. Conclui-se também que são necessários mais estudos, a fim de determinar em qual fase da reposta inflamatória a ação dos medicamentos ocorre: na diferenciação celular ou na síntese das citocinas. 


\section{REFERÊNCIAS}

AZIZI G.; JADIDI-NIARAGH F.; MIRSHAFIEY A. Th17 Cells in Immunopathogenesis and treatment of rheumatoid arthritis. Int. J. of Rheum. Diseas., v. 16, p. 243-253, 2013.

CAPETTINI L. S.; MONTECUCCO F.; MACH F.; STERGIOPULOS N.; SANTOS R. A.; DA SILVA R. F. Role of renin-angiotensin system in inflammation, immunity and aging. Cur. Pharm. Desg., v. 18, n. 7, p. 963-970, 2012.

DINH Q. N.; DRUMMOND G. R.; SOBEY C. G. CHRISSOBOLIS S. Roles of Inflammation, Oxidative Stress, and Vascular Dysfunction in Hypertension. Int. J. of Rheum. Diseas., v. 16, p. 243-253, 2013.

FURST D. E. e EMERY P. Rheumatoid arthritis pathophysiology: update on emerging cytokine and cytokine-associated cell targets. Rheumatology, v. 53, p. 15601569, 2014.

KOZERA L.; ANDREWS J.; MORGAN A. W. Cardiovascular risk and rheumatoid arthritis--the next step: differentiating true soluble biomarkers of cardiovascular risk from surrogate measures of inflammation. Rheumatology, v. 50, n. 11, p. 1944-1954, 2011.

LEE S. Y.; KWOK S. K.; SON H. J.; RYU J. G.; KIM E. K.; OH H. J.; CHO M. L.; JU J. H.; PARK S. H.; KIM H. Y. IL-17-mediated Bcl-2 expression regulates survival of fibroblast-like synoviocytes in rheumatoid arthritis through STAT3 activation. Arthritis Res. \& Ther., v. 15, p. R31, 2013.

MCMASTER W. G.; KIRABO A.; MADHUR M. S.; HARRISON D. G. Inflammation, Immunity, and Hypertensive End-Organ Damage. Circ Res., v. 116, p. 1022-1033, 2015.

SILVA A. C. S.; SILVEIRA K. D.; FERREIRA A.J.; TEIXEIRA M. M. ACE2, angiotensin-(1-7) and Mas receptor axis in inflammation and fibrosis. British J. of Pharm., v. 169, n. 3. p. 477-492, 2013.

SYMMONS D. P. M.; GABRIEL S. E. Epidemiology of CVD in rheumatic disease, with a focus on RA and SLE. Nature Rev. Rheumatol., v. 7, p. 399-408, 2011.

\section{AGRADECIMENTOS}

Este estudo foi apoiado pelo NUPIT-SG, CAPES, INCT_IF e CNPq. 